\title{
Balkanologie
}

Balkanologie Revue d'études pluridisciplinaires

Vol. I, n 1 | 1997

Volume I Numéro 1

\section{Continuité territoriale, continuité nationale}

L'exemple yougoslave

\section{Hervé Guillorel et Patrick Michels}

\section{CpenEdition}

\section{Journals}

Édition électronique

URL : http://journals.openedition.org/balkanologie/206

DOI : 10.4000/balkanologie.206

ISSN : 1965-0582

Éditeur

Association française d'études sur les Balkans (Afebalk)

\section{Édition imprimée}

Date de publication : 1 juillet 1997

ISSN : 1279-7952

\section{Référence électronique}

Hervé Guillorel et Patrick Michels, "Continuité territoriale, continuité nationale », Balkanologie [En ligne], Vol. I, $n^{\circ} 1$ | 1997, mis en ligne le 30 novembre 2007, consulté le 17 décembre 2020. URL : http:// journals.openedition.org/balkanologie/206 ; DOI : https://doi.org/10.4000/balkanologie.206

Ce document a été généré automatiquement le 17 décembre 2020.

(c) Tous droits réservés 


\section{Continuité territoriale, continuité nationale}

L'exemple yougoslave

Hervé Guillorel et Patrick Michels

\section{NOTE DE L'ÉDITEUR}

Cet article s'inscrit à la fois dans la logique des travaux de recherche menés depuis plusieurs années par Hervé Guillorel sur les rapports entre espace et politique, dans le cadre de l'I.P.I.E. et dans la logique du programme Mercator sur les minorités (en collaboration avec Henri Giordan). Ce texte est basé sur une communication d'H. Guillorel au Congrès Mondial de l'Association Internationale de Science Politique à Berlin en août 1994 (atelier Ethnicité et territorialité, direction : Jean Tournon).

1 Les processus d'édification étatique et surtout nationale ("state and nation-building") incluent systématiquement, au-delà de leurs individuations temporelles et spatiales, un bricolage idéologique dont la fonction première est d'affirmer la continuité d'une "communauté" nationale préexistante, incréée, "déjà-là", d'où une vision a-temporelle et linéaire de l'histoire prouvant, au-delà des ruptures et des discontinuités, la permanence d'une entité nationale qui transcenderait tous les autres clivages et notamment les clivages sociaux ${ }^{1}$ : cette continuité peut être historique, linguistique, territoriale, ethnique, juridique, archéologique, etc. ${ }^{2}$ Nous avons ici un cas classique de "réification".

2 Que l'on parle de mythe, d'invention, de reconstruction et/ou d'imagination ("invention de la tradition", "communautés imaginées", invention des continuités historiques, etc.), on retrouve toujours des questionnements qui tournent autour de remarques épistémologiques sur la dialectique continuité/discontinuité ${ }^{6}$. Nous pouvons reprendre l'affirmation du linguiste B. Cerquiglini : «Cette question du continu et du discontinu est une des apories de la linguistique historique $»^{7}$ et la généraliser à toute démarche historisante voire à toute démarche scientifique. 
3 Cette invention de la tradition suppose également une quête des origines et l'élaboration de mythes fondateurs (héros, peuples, dynasties, langues, batailles, berceaux historiques pour ne pas dire préhistoriques $\left.{ }^{8}\right)$. On peut interpréter cette démarche comme une ré-écriture, une ré-appropriation, une falsification ou une décolonisation de l'histoire, selon les époques, selon les points de vue, etc. C'est ainsi que, par exemple, les anciennes colonies, les minorités nationales ou les sociétés postcommunistes re-découvrent et ré-écrivent leurs "histoires nationales".

4 Comme le dit très bien $\mathrm{B}$. Melman dans un article sur "l'invention de la tradition anglosaxonne" :

La reconstitution des continuités d'une histoire appropriée qui relie le présent au passé, caractérise la plupart des sociétés lors des phases de transition. Seul le passé, a remarqué Disraeli, donne de l'énergie à une race antrophiée lorsque tout le reste n'y est pas parvenu. Un passé inventé, prudemment manipulé, non seulement “explique le présent", mais "façonne le futur". Les continuités inventées, pour paraphraser l'expression très utilisée d'Hobsbawm, sont plus à même de se développer dans les communautés modernes. En fait, la quête des continuités historiques doit être principalement recherchée en ces lieux et ces temps où l'identité nationale émerge et se cristallise.

Mais ce ne sont pas seulement les nations nouvelles ou immatures, ou les groupes cherchant à s'attacher à une "nation" réelle ou imaginée, qui légitiment l'innovation par l'invention d'une tradition. Dans certaines communautés nationales les plus vieilles, telles que l'on peut les retrouver dans les États-nations "matures", les identités ont constitué un défi, définies et redéfinies dans divers processus d'inclusion [dans la nation], d'exclusion [hors de celle-ci] et de transformation. ${ }^{9}$

5 Les situations de transitions, voire de crises, sont un terreau favorable pour analyser ces processus. Le but de cet article est de proposer quelques pistes de recherche, sous la forme d'un début de typologie, pour analyser comment les mythes / histoires nationalistes (nationalitaires, régionalistes, etc.) ${ }^{10}$ ont présenté et présentent toujours, une vision de la manière dont se sont formées les "entités" qu'ils postulent, notamment leurs origines ethniques ${ }^{11}$.

6 Nous estimons que cette problématique idéologique est un passage obligé de toute perspective nationaliste. Simplement, compte tenu des caractères plus ou moins éloignés et du succès (passage de l'ethnos au demos) ou de l'échec des processus d'édification étatique et nationale, cette problématique peut être aujourd'hui oubliée et peut devenir, provisoirement ${ }^{12}$, non-pertinente, mais elle peut également être totalement ré-actualisée et opératoire dans les États-nations en voie de structuration (tiers-monde, éclatement des Empires, etc.). Cette problématique est donc axée autour du problème de l'“ethnogénèse".

7 Le Dictionnaire de l'ethnologie et de l'anthropologie dirigé par P. Bonte et M. Izard ne cite le terme ethnogénèse que pour effectuer un renvoi à l'entrée intitulée: URSS - Les anthropologues russe et soviétique ${ }^{13}$. En effet, "la notion d'ethnos a été introduite en 1923 par S. M. Shirokogoroff pour désigner non seulement ce que nous entendons par culture mais aussi ce que nous appellerions l'ethnicité ou l'identité ethnique »: la démarche est d'inspiration historiciste et met l'accent sur les processus d'ethnogénèse. C'est une démarche que l'on retrouve dans les productions des ex-pays de l'est ${ }^{14}$.

8 L'expression ethnogénèse se retrouve plus rarement dans le cas de l'histoire yougoslave $^{15}$ : cette absence peut être expliquée justement par la complexité du "pluralisme ethnique" dans ce pays, alors qu'en Roumanie, en Hongrie ou en Albanie, 
l'ethnogénèse renvoie à la dynamique d'un "noyau dur", homogène, notamment par son contraste avec un environnement slave.

9 Aujourd'hui, le terme "ethnogénèse" se développe au-delà de son contexte originel et est utilisé pour décrire de nombreuses sociétés pluri-ethniques, notamment dans les anciennes "colonies". Des travaux récents sont centrés sur la question de l'ethnogénèse considérée comme l'étude dynamique des impacts des migrations, des conflits et autres processus de multiplication des "contacts" entre groupes, sur les renforcements voir même la genèse des identités communautaires ${ }^{16}$. Les processus d'ethnogénèse sont étudiés par de nombreuses disciplines: la linguistique historique ${ }^{17}$, l'archéologie, les études folkloriques, l'histoire, la géographie, l'ethnologie, etc.

Cette pluridisciplinarité nous a incité à prévoir une première partie consacré à l'étude de ce que nous appellerons la mobilisation/manipulation différentielle des disciplines. Elle aura pour finalité de montrer que les idéologies nationalistes (régionalistes, nationalitaires, etc.) sont forgées par des intellectuels qui occupent souvent des positions scientifiques dans les différents champs du savoir.

11 Notre seconde partie, plus longue, propose un essai de typologie des contacts interethniques basé sur les processus réels et / ou imaginés caractérisant l'ethnogénèse ou l'histoire ethnique. Cette typologie sera illustrée par des exemples empiriques très divers tirés de contextes historiques et spatiaux très différents, avec une préférence pour l'Europe Centrale et Orientale.

Dans les deux parties, nous analyserons en détail en quoi cette typologie peut être illustrée en tout ou partie à l'aide du cas yougoslave (ici le mot "yougoslave" renvoie à l'espace yougoslave correspondant à l'État créé en 1918 et démantelé en 1991), bien que nous insisterons sur le cas serbe, le peuple le plus diasporique au niveau infrayougoslave.

\section{La mobilisation/manipulation différentielle des disciplines}

Dans une contribution consacrée aux "Albanais et Serbes en Yougoslavie", W. Vermeer écrit :

Dans la plupart des pays, les universitaires intéressés par la passé (historiens, linguistes historiques, ...) se trouvent en dehors de la société. Ce qu'ils font peut conjoncturellement être intéressant, mais est aussi éloigné des vrais problèmes de la société que le concerto pour clarinette de Mozart (KV 622). En Yougoslavie, les choses sont un peu différentes. Là, la société attend des historiens qu'ils fournissent des preuves "scientifiques" des prétentions territoriales. La société serbe attend de l'historien qu'il prouve que la Serbie a le droit d'occuper le Kossovo, même si aujourd'hui les Serbes ne constituent qu'une minorité de sa population. ${ }^{18}$

14 Et quelques pages plus loin, le même auteur écrit : «il est troublant de voir que même une institution comme l'Académie serbe, dont la finalité première devrait être la vérité, n'hésite pas à manipuler la réalité historique pour se conformer aux demandes politiques de la société $»^{19}$.

15 Ces deux propos témoignent d'une certaine naïveté quant au rôle des intellectuels et des organismes "scientifiques" dans l'élaboration des idéologies nationalistes. Leur implication manifeste ou latente a été et est la règle dans de nombreux pays; à cet égard la Yougoslavie et la Serbie ne font pas exception. On peut seulement avancer 
l'hypothèse que cette mobilisation des intellectuels ("organiques") est en partie déterminée par les caractéristiques de la société (société globale mais aussi sociétés régionales ou locales) dans laquelle ils sont immergés (sociétés en crise, en transition, notamment au regard des processus d'édification étatique et nationale $)^{20}$. Cela permet d'effectuer des comparaisons entre des sociétés très éloignées dans le temps et dans l'espace. La Yougoslavie, à cet égard, cumule des "handicaps" : fragmentation ethnique, linguistique et religieuse, caractère très tardif du processus d'édification étatique et nationale, hégémonie communiste pendant plus de quarante ans. Comme le fait remarquer St. K. Pavlowitch dans le compte-rendu récent des Essais en l'honneur de Dimitrije Djordjevic,

[Ce] recueil ne serait pas complet sans une contribution qui montre, malheureusement, que Clio, après avoir réussi à préserver une grande partie de sa capacité critique sous l'épuration communiste, est en danger de s'effondrer avec le communisme. Un historien serbe distingué a trouvé le moyen d'écrire sur l'exceptionnalité du destin historique qui distingue le peuple serbe des autres nations européennes, en particulier de leurs voisins, dans un recueil en l'honneur d'une approche toujours critique de l'histoire des Balkans. ${ }^{21}$

Dans ce processus idéologique, toutes les disciplines peuvent être mobilisées : l'histoire, en premier lieu, car comme l'affirmait au siècle dernier un historien nationaliste breton, "l'histoire est donc, à la lettre, la science patriotique par excellence $»^{22}$, mais aussi la géographie, la linguistique, l'archéologie et l'ethnologie, pour ne citer que les cas les plus intéressants. Cette mobilisation de disciplines très diverses et dont la technicité est de plus en plus complexe implique que toute tentative de "déconstruction" des idéologies nationalistes soit faite dans une perspective pluridisciplinaire.

On peut citer, par exemple, la complexité technique de la question macédonienne: l'importante surdétermination politique, idéologique et affective actuelle de cette question ne peut pas être simplement affirmée puis écartée. Il est nécessaire de traquer ces surdéterminations y compris dans les moindres "détails techniques".

Selon le "professeur" bulgare D. Gocev, dans un article consacré au rôle des érudits bulgares au sein du mouvement national de libération des Bulgares de Macédoine à la fin du $\mathrm{XIX}^{\mathrm{e}}$ siècle et au $\mathrm{XX}^{\mathrm{e}}$ siècle, "les universitaires bulgares actuels et toute notre intelligentsia a une grande leçon à apprendre de ses prédécesseurs. Ils doivent, dans leurs activités scientifiques et publiques, d'une manière digne, défendre les problèmes nationaux bulgares et élever la nouvelle génération dans un esprit de patriotisme, d'amour et de loyauté envers leur peuple et leur patrie ${ }^{23}$. La mission des intellectuels bulgares est donc toute tracée.

Cet article est paru dès la réapparition, à Sofia, de la Macedonian Review, en 1991, présentée de la façon suivante : « une tribune stable est restorée qui, avec le pouvoir de la science, défendra la vérité à propos des Bulgares de Macédoine, prouvera leur identité nationale et le caractère de leurs luttes épiques pour l'indépendance culturelle et politique, soutiendra l'esprit national bulgare dans ce territoire historiquement bulgare qui a tant souffert $»^{24}$. "Pouvoir de la science", "défendre la vérité", "prouver leur identité nationale", "luttes épiques", "soutenir l'esprit national", "le territoire bulgare historique" : nous retrouvons bien ici les ingrédients "classiques" de l'idéologie nationaliste ${ }^{25}$. ouvrage récent consacré à l'émergence de la Macédoine, l'universitaire américain $\mathrm{E}$. $\mathrm{N}$. 
Borza estime que l'ouvrage de N. K. Martis ${ }^{26}$ constitue la version la plus choquante de la position chauvine grecque : «ce livre écrit par un ancien Ministre du nord de la Grèce est un pamphlet anti-yougoslave, contenant tant d'erreurs et de distorsions historiques que le prix que lui a décerné l'Académie d'Athènes ne sert qu'à mettre en doute la confiance dans le jugement scientifique de cette vénérable société d'érudits ${ }^{27}$.

Ainsi, l'Académie Serbe et l'Académie d'Athènes, sans oublier la Macedonian Review, "revue scientifique" sont à mettre dans le même panier.

Ces exemples tirés de la "question macédonienne" posent le problème général des biais nationalistes: l'universitaire américain $\mathrm{H}$. Lunt a récemment abordé cette question dans le domaine des études slaves. Selon lui, « dans les années 1990 nous devons porter une attention particulière aux jugements de valeur associé à des attitudes nationalistes, en particulier aux présuppositions fondées sur les enseignements de l'ensemble nationaliste ${ }^{28}$. En effet, « de profondes émotions se cachent dans les questionnements universitaires sur les origines ethniques: la revendication implicite que mes ancêtres sont meilleurs que les vôtres est un écueil dangereux qui n'est pas toujours apparent sous les eaux agitées des disputes académiques ${ }^{29}$. Et il conclut de la façon suivante : "aussi difficile que cela soit, les slavistes doivent continuellement surveiller leurs propres perceptions des préjugés nationalistes (et autres) $»^{30}$.

Il est vrai qu'H. Lunt, spécialiste de la Macédoine, se devait d'être particulièrement attentif à ces questions et ne pouvait qu'être la cible privilégiée d'une majorité des acteurs en présence (Grèce, Bulgarie, Serbie, République Yougoslave de Macédoine). Mais son avertissement ne doit bien sûr pas être cantonné aux "slavistes" mais être universalisé. La mobilisation / manipulation différentielle des disciplines peut être analysée de diverses manières : selon bien sûr les disciplines mais également selon les publics et ici il faut faire une place importante au rôle des manuels scolaires comme instruments fondamentaux de socialisation nationaliste.

C. Jelavich a étudié les biais nationalistes dans les publications serbes avant 1914 et notamment dans les manuels scolaires, de géographie, d'histoire et de littérature. Les manuels serbes de géographie ont été fortement influencés par les écrits d'un auteur, le professeur Vladimir Karić. Cet auteur affirme que tous les peuples qu'on appelle aujourd'hui slaves étaient autrefois appelés serbes et que les Slaves n'étaient qu'une tribu serbe. Les cartes des populations de l'Europe du sud-est reproduites dans ses ouvrages identifient bien les Serbes, les Slovènes, les Allemands, etc., mais oublient les Croates. La Croatie, la Slavonie, l'Istrie, la Dalmatie, la Bosnie-Herzégovine, la Bačka et le Banat sont représentés comme n'étant habités que par des Serbes.

Les manuels d'histoire reproduisent les mêmes biais. Certains d'entre eux ont été écrits par d'éminents universitaires de l'Université de Belgrade (ayant été en partie ministres de l'Éducation et / ou membres de l'Académie serbe). Selon eux, tous les Slaves à une époque furent appelés Serbes, et en 1900, l'un d'entre eux écrit que les Serbes ont conquis et occupé les territoires suivants : Banat, Bačka, Srem, Slavonie, Croatie, Istrie, Dalmatie, Bosnie, Herzégovine, Monténégro, Serbie, Vieille Serbie (Kosovo), Macédoine et les parties occidentales de la Bulgarie et de la Roumanie actuelles.

27 C. Jelavich conclut en montrant que, même si les versions de ces manuels serbes datant de 1913 et 1914 commencent à accorder une petite place au thème des Slaves du Sud 
(des Yougoslaves), ils arrivèrent trop tard pour avoir une influence significative sur les lecteurs serbes.

Ces manuels nous montrent comment on enseignait aux Serbes la façon de percevoir leur nation et qu'ils ne connaissaient que peu les autres Slaves du sud, dont le destin sera lié au leur. Ces manuels scolaires d'avant-guerre nous aident aussi à expliquer le tour malheureux que les affaires sud-slaves prirent dans l'entredeux-guerres, voire les tragiques événements de la Seconde Guerre mondiale. ${ }^{31}$ être appréhendés dans un contexte historique particulier, celui des peuples serbes. Il est né en 1787 et mort en 1864. La Serbie a obtenu son indépendance quelques années après sa mort. Karadžić appartient à la phase préparatoire de l'indépendance, la phase politico-intellectuelle connue sous le nom de "question orientale". Cette période fut caractérisée par un renouveau de l'intérêt pour la langue et par un certain nombre de travaux littéraires, essentiellement de type ethnographique, travaux qui sont significatifs de l'histoire littéraire des peuples à la recherche de leur indépendance. La recherche d'épopées nationales mythiques et historiques, transmises par les vecteurs de ballades épiques et autres chants populaires transmis oralement de générations en générations se retrouvent, au xix ${ }^{\mathrm{e}}$ siècle, dans de nombreux cas, qu'il s'agisse d'Ossian pour l'Écosse, du Barzaz Breiz pour la Bretagne ou du Kalevala pour la Finlande, pour ne citer que quelques cas. De nombreux auteurs ont souligné l'importance fondamentale des chants épiques serbes comme vecteurs de la continuité nationale du peuple serbe. Voici par exemple ce qu'écrit R.W. Seton-Watson :

Ainsi de 1459 à 1804 la Serbie a cessé d'exister en tant qu'État et nation. Comment se fait-il qu'elle fut capable de revenir d'entre les morts? Il n'y a probablement pas d'autre exemple dans l'histoire d'une nation qui s'est préservée par sa poésie nationale. Cela semble difficilement possible, et pourtant c'est littéralement vrai en ce qui concerne les Serbes. Chaque Serbe est à moitié poète, et lorsque tout semblait perdu, les bardes locaux ou les poètes gardèrent vivace la mémoire des gloires passées de la Serbie par leurs chansons ou leurs ballades, et chantèrent aussi les glorieux jours anciens qui reviendraient à nouveau et les consoleraient des misères du présent. ${ }^{32}$

31 Et l'auteur rappelle que les plus belles ballades et les plus émotives sont centrées sur les événements de la bataille de Kosovo. "Il est difficile pour nous de comprendre comment l'histoire de Kossovo est complètement liée à la vie quotidienne de l'entière nation serbe $»^{33}$.

Préfaçant un recueil en français de ballades serbes sur la bataille de Kosovo, M. R. Vesnić, ministre du tout nouveau "Royaume-Uni des Serbes, Croates et Slovènes", écrit que «Kossovo est la synthèse de toute l'histoire du peuple serbe $»^{34}$. À ce propos, Albanais et Serbes, pour prouver leur supériorité numérique à l'époque ottomane, et donc un droit historique sur la région, avancent des chiffres fondés sur des recensements ottomans ${ }^{35}$, tandis que du côté albanais on évoque le cadastre vénitien de Shkodër / Skadar de $1416^{36}$; l'archéologie aussi est utilisée, d'un côté les nombreux monastères serbes $^{37}$, de l'autre des traces de civilisation illyrienne et albanaise 
"confirmant" leur apparentés ${ }^{38}$, comme en Macédoine ${ }^{39}$, toutes sortes de cartes ethnographiques ont été avancées, se modifiant au gré des circonstances. Par exemple, Cvijić augmente la composition serbe de la Macédoine après l'annexion de la BosnieHerzégovine par l'Autriche-Hongrie (1908) et sur les côtes du lac Scutari / Skadar ${ }^{40}$, la thèse de l'albanisation serbe explique aussi que tous les Albanais ne doivent pas être comptés comme albanais, ce qui expliquerait la diminution du nombre d'Albanais dans les cartes de Cvijić.

L'étude comparée des chants populaires, des coutumes, des traditions, ce qu'on pourrait appeler la science folklorique va par la suite être plus scientifiquement organisée et aboutir à l'ethnologie.

L'histoire de l'ethnologie en Europe de l'est montre l'existence d'un rapport étroit entre une "science" des coutumes nationales et la demande politique visant à définir et instaurer une continuité "nationale" ${ }^{41}$. "Un des fondements sur lesquels l'ethnologie s'est constituée comme science en Serbie, est l'histoire et la philologie. L'historiographie et la philologie serbes exercèrent une forte influence sur l'ethnologie pour qu'elle forme une étude nationale de la nation $»^{42}$. Pavković, Bandić et Kovačević identifient plusieurs approches: l'orientation "anthropogéographique" étudie les relations et les influences mutuelles entre la nature, l'homme et la société ${ }^{43}$. Le fondateur de cette orientation est Jovan Cvijić et les résultats de cette méthode sont très bien présentés dans l'édition Settlements and the origin of the population (Miscellany of Serbian Academy).

De son côté, la Matica srpska proclame que l'étude de l'origine de la population et des coutumes populaires constitue sa tâche la plus importante. La recherche doit être faite de manière à ce que les régions soient prises comme entités séparées, sous la forme de monographies ethnologiques et avec un intérêt particulier sur les différents restes des autres nations entrant dans la composition et la culture ethniques des habitants d'aujourd'hui ainsi que sur certains processus anciens de colonisation et les établissements du $\mathrm{xx}^{\mathrm{e}}$ siècle ${ }^{44}$.

Nous avons mentionné plus haut le nom de Jovan Cvijić. Cet universitaire présenté tantôt comme géographe, tantôt comme ethnologue ou anthropologue, a joué, avec son équipe de collaborateurs, un rôle fondamental ${ }^{45}$. Dès la fin du XIX ${ }^{e}$ siècle, Cvijić a initié des séries anthropologico-géographiques de monographies qui recherchent les origines et les mouvements migratoires des populations de régions particulières.

Les travaux développés par cette "école" ont été utilisés dans le mémorandum présenté lors de la Conférence de la Paix à Versailles en 1918, lors de l'établissement de l'État yougoslave. En effet, le principe des nationalités a joué un rôle vital dans la définition des frontières de ce nouvel État. Il était convenu que les critères de ces frontières devraient être "ethnographiques"

Jovan Cvijic, par exemple, l'ancien recteur de l'Université de Belgrade, devint directeur de la section géographique et ethnographique de la délégation. Ses fonctions étaient en fait stratégiques. Géographe balkanique réputé en Europe, Cvijic devint effectivement l'arbitre de plusieurs contestations techniques que provoquèrent au sein de la délégation les demandes yougoslaves au nord, au nordest et à l'est, qui portaient indubitablement sa marque. ${ }^{47}$

Un autre universitaire a joué également un rôle important, il s'agit de T. R. Georgevitch, auteur de fascicules et d'ouvrages cherchant à prouver le caractère serbe de nombreuses populations situées à la périphérie de la Serbie historique (Macédoine, 
Bulgarie, etc.). Il est intéressant de noter ici, parmi les arguments avancés, celui de la connaissance par les populations concernées des ballades relatives à la bataille de Kosovo et aux héros serbes traditionnels ${ }^{48}$.

Les exemples mentionnés ci-dessus montrent bien de quelle manière les intellectuels ont été / se sont mobilisés pour illustrer et défendre la continuité et l'unicité de leur entité nationale. Cette mobilisation affecte toutes les disciplines et tous les vecteurs de diffusion. Dans un ouvrage récent sur la "destruction de la Yougoslavie", B. Magaš intitule l'un de ses chapitre "nationalism captures the Serbian intelligentsia $»^{49}$. Il est vrai que de nombreux auteurs accusent Slobodan Milošević d'avoir été le premier à réaliser que le communisme était mort en Yougoslavie et surtout d'avoir été le premier qui a plus ou moins ouvertement opté pour la stratégie nationaliste : «En exploitant la "question nationale serbe" comme principale ressource, Milošević a promu une politique typiquement totalitaire, centrée autour du mythe de l'unité nationale et soutenue par une constante fabrication d'ennemis contre lesquels "nous devons nous unir" $»^{50}$.

Mais les auteurs de l'article dont est extrait cette citation étendent leurs accusations de nationalisme aux autres dirigeants des diverses Républiques de l'ex-Yougoslavie, et dans un chapitre intitulé « Rise of the tribal chiefs ", écrivent :

Les promoteurs de la dissolution de ce pays peuvent être appelés "les nationalistes associés de la Yougoslavie" (Milosevic, Tudjman et Kucan étant, parmi eux, les plus importants). Ces nouveaux dirigeants et leurs partisans se sont radicalement opposés à la reconstruction démocratique de la Yougoslavie, parce qu'ils savaient qu'une telle reconstruction ébranlerait le fondement de leur pouvoir. Bien qu'élus (relativement) démocratiquement, les présidents (exceptions faites du président macédonien Gligorov et, jusqu'au début de la guerre en Bosnie, de son homologue bosniaque Izetbegovic) ont fondé leur pouvoir sur la manipulation des préjugés nationalistes irrationnels... Le monisme socialiste fut simplement éclipsé au profit d'une sorte de quasi-pluralisme nationaliste. ${ }^{51}$

\section{Essai de typologie : du désert à la fusion ethnique}

Dans un article récent consacré aux conflits ethniques dans les îles Fidji et Maurice, $\mathrm{T}$. Carroll s'intéresse aux prétentions et revendications basées sur un sentiment d'“autochtonéité" (qu'il appelle "indigenousness") et écrit : « il est surprenant (...) que peu d'études comparatives examinant explicitement le caractère et les conséquences de l'affirmation de l'“autochtonéité" soient publiées " $^{52}$.

Pourtant cette prétention à l'autochtonéité est un sentiment d'appartenance, de légitimité territoriale que l'on trouve très fréquemment, à de nombreux niveaux, qu'il s'agisse de prétentions territoriales avancées par des États et concernant des espaces qui peuvent être très vastes, ou des sentiments de possession portant sur des microespaces, tant en milieu rural qu'en milieu urbain. Le mot "autochtone" renvoie aujourd'hui, dans un sens restreint, aux idéologies et luttes tant politiques que juridiques menées par des "peuples" tels que les Indiens d'Amérique, les Aborigènes d'Australie ou les Saamis, dans un cadre international ou dans un cadre interne (ÉtatsUnis d'Amérique, Canada, etc.) : les termes "aborigène", "natif", "endogène" sont des synonymes. Ces qualificatifs s'appliquent tant à des peuples ou ethnies qu'aux langues qu'ils parlent. Généralement, on réserve ces expressions à des communautés "éloignées 
dans le temps et dans l'espace" des peuples qui les ont "colonisés", notamment en provenance de l'Europe.

Dans le cadre de notre travail, nous avons élargi la notion: nous appelons "autochtones" les groupes ou les ethnies qui étaient "déjà là" lors de contacts ultérieurs avec des peuples migrateurs.

Bien évidemment, l'analyse des récits de fondation témoignent de l'inexistence de véritables autochtonies dans la mesure où ils font presque tous état de mythes fondateurs, donc de migrations fondatrices. Simplement, ces migrations fondatrices peuvent impliquer diverses modalités de contacts inter-ethniques, sauf le cas exemplaire et souvent fantasmé ou reconstruit du désert.

Mais les thèses de l'autochtonie comme les thèses migrationnistes, au-delà de leur divergence portant sur le point de savoir qui était le premier à tel endroit, s'accordent sur l'importance à accorder à la notion de continuité, continuité prouvée et reconstruite par tous les moyens, y compris par le cumul temporel et/ou spatial de discontinuités.

Au tout début de La guerre du Péloponnèse, l'historien grec Thucydide écrit : « Il est clair que le pays appelé aujourd'hui Hellade n'était pas autrefois habité de façon stable. Il fut à l'origine le théâtre de migrations et les populations abandonnaient sans résistance les terres qu'elles occupaient, sous la pression de nouveaux arrivants qui se trouvaient être chaque fois plus nombreux $»^{53}$. Mais il écrit plus loin :

C'est à coup sûr à l'aridité de son sol que l'Attique doit d'avoir, aussi loin que l'on remonte dans le passé, ignoré les conflits internes et conservé au cours des âges la même population. L'observation suivante prouve bien que c'est à cause des migrations que les autres contrées ne connurent pas un développement équivalent : parmi ceux que les guerres civiles ou étrangères chassaient des autres pays, les plus riches se réfugiaient à Athènes, où ils comptaient trouver un asile sûr. Devenus citoyens athéniens, ils grossirent dès les temps anciens la population, au point que, par la suite, les ressources de l'Attique devenant insuffisantes, on envoya des colonies en Ionie..$^{54}$

47 Ces deux passages de Thucydide présentent en même temps les thèses migrationnistes pour l'ensemble de la Grèce et la thèse de l'autochtonie au seul profit d'Athènes : nous verrons plus loin d'autres cas empiriques où s'affrontent (dans le temps et / ou dans l'espace) les thèses migrationnistes et les thèses de l'autochtonie.

\section{Typologie}

(i) L'autochtonie "originelle" ou la continuité ethno-territoriale (continuité ethnique dans le même lieu) : c'est l'exemple précité de l'Attique. C'est également la thèse proposée par les historiens roumains pour justifier la continuité daco-romaine (puis roumaine) pendant plus de 2000 ans sur le territoire actuel de la Roumanie. Cette question de l'ethnogénèse roumaine et de la "continuité" latine en Europe du sud-est est dotée d'une charge politique et affective très forte porteuse de préjugés nationalistes, voire chauvins, très répandus parmi les intellectuels roumains et hongrois.

49 En effet, depuis longtemps les avis sont partagés au sujet du lieu d'origine du peuple roumain. L'historien autrichien Roesler avait, en 1871, tenté de démontrer la thèse selon laquelle les Roumains ne descendent pas de la population romanisée de Dacie, mais ont émigré beaucoup plus tard à partir des régions situées au sud du Danube : 
cette thèse "migrationniste", ou de la "continuité mobile", a trouvé beaucoup d'adeptes chez les savants hongrois, pour des raisons politiques et idéologiques puisqu'elle “prouvait" l'antériorité de l'établissement hongrois. À cette thèse migrationniste s'oppose la théorie de la "continuité" territoriale ou endogène, ou de l'autochtonie, thèse qui a toujours été défendue par la presque totalité des savants roumains.

La Transylvanie joue un rôle central dans les histoires et les légendes "nationales" de la Hongrie et de la Roumanie. Dans les deux cas, elle est perçue comme le berceau de leur culture et de leur civilisation. Dès lors, le conflit principal se focalise sur la question : qui était là le premier?

51 La version hongroise affirme que les Magyars de Transylvanie appartenaient à la migration des Magyars qui s'établirent au $\mathrm{x}^{\mathrm{e}}$ siècle, en provenance d'une région située entre la partie moyenne de la Volga et les montagnes de l'Oural. Ils occupèrent la plaine de Pannonie et la Transylvanie, régions qu'ils trouvèrent largement dépeuplées. Selon cette version, les Roumains arrivèrent plus tard: ce n'est qu'au xiII siècle que les nomades et les bergers roumains traversèrent les Carpathes et que les Hongrois leur accordèrent généreusement le droit de s'établir.

52 La version roumaine est très différente. Les Roumains prétendent que les Szeklers (Sicules) étaient à l'origine des Roumains qui avaient été forcés de devenir Hongrois. Selon l'histoire officielle roumaine, la Transylvanie fut le noyau de l'Empire Dace. Ce territoire fut ensuite intégré au sein de l'Empire Romain, au II $^{\mathrm{e}}$ siècle après J.C. La culture nationale roumaine est le résultat d'une fusion des cultures dace et romaine. Les Romains abandonnèrent la province de Dacie en 271, mais la Transylvanie demeura un carrefour de migrations pour plus de 700 ans. Les Daces romanisés n'ont absolument pas quitté leur territoire mais ont dû se cacher dans les forêts et dans les montagnes, assurant ainsi la vitalité de la culture roumaine. Cette théorie d'une continuité dacoromaine de plus de 2000 ans justifie donc que la Transylvanie appartienne aux Roumains car elle confirme leur primauté en tant que population autochtone.

Dans le cas de la Roumanie, l'usage de l'autochtonie est encore plus complexe: par exemple, dans un article récent consacré à l'historiographie roumaine, Dennis Deletant montre comment, dans les années 1980, les ré-écritures de l'histoire effectuées par Ilie Ceauşescu, vice-ministre de la Défense, ont cherché à diminuer le rôle de la romanité et à renforcer celui des autochtones Daces, dans la Transylvanie actuelle, de manière à légitimer la politique de son frère Nicolas Ceausescu, "Condutatore" de la Roumanie communiste ${ }^{55}$.

54 Pour finir avec le cas complexe de la Transylvanie, il faut encore mentionner une théorie proposée au $\mathrm{xvI}^{\mathrm{e}}$ siècle qui prétendait que les Daces, les premiers habitants historiques de la Roumanie, étaient une tribu germanique. Il était également connu que d'autres peuples germaniques de l'Antiquité tardive, tels que les Goths ou les Gépides, avaient vécu dans cette même partie de l'Europe avant l'invasion des Huns et d'autres nomades de la steppe. L'hypothèse proposée était que les Saxons de Transylvanie constituaient les restes de ces populations germaniques installées beaucoup plus tôt, et que leur établissement en Transylvanie précédait non seulement celui des Magyars mais même celui de la Rome impériale. C'est ainsi qu'en 1591, un comte saxon a pu évoquer cette "théorie de la continuité" pour défendre la position saxonne, à l'aide d'un argument qui annonce les prétentions affirmées des siècles plus tard par les Roumains. En réalité les Saxons de Transylvanie ont été implantés, dans le cadre d'un 
processus de "colonisation" dirigé par la royauté hongroise désireuse de mieux défendre une frontière orientale fragile.

Le cas albanais est à certains égards proches du cas roumain par le souci des historiens de ces deux pays de vouloir à tout prix prouver la thèse de la continuité ethnique (de l'autochtonie) sur leur territoire actuel. Dans un ouvrage récent qui tente de faire le point sur la question, Gottfried Schramm, se prononce à la fois contre la continuité de l'ethnie albanaise dans son pays d'aujourd'hui (mais pour une continuité albanaise dans la région du Kosovo et de Dukagjin) et contre la continuité de l'ethnie roumaine en Dacie, heurtant ainsi de front les thèses officielles autochtones "isolationnistes" des deux communautés. Selon lui, l'ethnie et la langue roumaines se sont formées au sud du Bas-Danube et de la Save: il s'agit d'une deuxième romanitée ${ }^{56}$, d'une "romanité pastorale", mobile, résultant du fait, qu'après l'écroulement de la frontière nord de l'Empire Romain, la majorité de la population romanisée de la plaine du Bas-Danube avait cherché refuge dans les régions montagneuses ${ }^{57}$.

Il y a un parallèle étroit entre la situation roumaine et la situation albanaise et c'est pour cela qu'A. Ruzé, dans un ouvrage très critiqué et au titre éloquent, s'efforce de minimiser à tout prix les rapports entre le roumain et l'albanais : en effet, ce faisant, il se trouve d'accord avec la plupart des chercheurs roumains et albanais qui, soutenant l'hypothèse de la continuité autochtone de leurs deux peuples (Daces + /- romanisés è Roumains et Illyriens è Albanais), ont conscience que cette hypothèse serait gravement infirmée par l'existence de correspondances trop étroites entre le roumain et l'albanais ${ }^{58}$.

57 Le même auteur cherche également à minimiser la part du slave et du hongrois dans la toponymie roumaine.

(ii) Fantasme, mythe, hypothèse et / ou argument du "désert" : par définition l'hypothèse du désert implique une migration fondatrice.

(iia) désert

- désert "vrai":

Une ethnologue travaillant en Afrique cite les propos suivants relatifs à des récits de fondation : «Aux origines, disent-ils, il n'y avait rien ici. Chassés d'un royaume où notre soeur, celle qui nous guidait - la sarawniya, reine-prêtresse - devait être mise à mort, nous sommes arrivés au bord de cette vallée. Nous avons trouvé une pierre habitée par un génie du nom de Tunguma qui nous a dit de rester là. Ainsi nous avons donné souche aux habitants de la vallée $"^{59}$.

L'insularité peut constituer également un cas idéal.

- désert "humain" car les autochtones ne sont pas considérés par les arrivants comme des humains.

(iib) quasi-désert

- débris de populations

Les hypothèses du désert ou du quasi-désert sont nombreuses. Ainsi, dans un ouvrage publié en 1940 pour célébrer l'annexion de la Ruthénie par la Hongrie en 1939, le savant hongrois S. Bonkalo affirme que, jusqu'au XIII siècle, la région subcarpathique était inhabitée et formait un "no-man's land" protégeant le royaume hongrois. L'implantation de Ruthènes se serait effectuée à la suite de l'invasion mongole de 1241-1242. Cette thèse du désert est fortement contestée car il y a des preuves d'établissements humains postérieurs à la conquête hongroise. D'autre part, la plupart des noms de rivière dans la région de Bereg (Beregovo) ont une origine slave. Ici, la 
thèse du désert va de pair avec l'idée d'un établissement lent et pacifique (colonisation) commandé par le pouvoir hongrois ${ }^{60}$.

(iii) Dans l'hypothèse du non-désert, on peut ensuite procéder à une typologie des contacts inter-ethniques :

- génocide

- repousser (dans les montagnes, à la mer, dans les villes, etc.) : on retrouve ici les questions très importantes du clivage rural/urbain et du clivage nomade/ sédentaire ${ }^{61}$

- assimilation et / ou fusion (suivant les rapports de force)

- déclassement des "autochtones" (sous forme de castes inférieures)

- non-reconnaissance des autochtones : dans de nombreux cas, les autochtones ne sont pas reconnus en tant que tels, c'est-à-dire comme "déjà-là" (sans pour autant être les premiers) avant les groupes dominants, mais sont transformés en tribus arriérées de ces mêmes dominants. Ici les exemples abondent : les aborigènes en Inde, les Kurdes appelés "Turcs de la montagne". De la même façon, les Allemands implantés en Haute-Silésie et incorporés dans le nouvel État polonais en 1918 se sont vu parfois refuser leurs droits de minorités (notamment linguistiques) sous le prétexte qu'il ne s'agissait pas vraiment d'Allemands mais de Polonais "germanisés". L'étude des relations géopolitiques entre ces groupes permettrait d'affiner la typologie: ainsi, les Pomaks, musulmans bulgarophones de Bulgarie, sont considérés par le gouvernement d'Ankara comme des "Turcs bulgarisés" et par le gouvernement de Sofia comme des "Bulgares islamisés".

- problème de la reconnaissance des autochtones comme "peuples fondateurs" ou "constitutifs" des États au sein desquels ils vivent. C'est par exemple la revendication des Crees au Québec.

61 Ces différentes variantes des processus d'ethnogénèse peuvent être illustrées par de nombreux $\operatorname{cas}^{62}$, l'exemple yougoslave est intéressant de par l'importance fondamentale accordée aux processus de migration.

\section{Le cas yougoslave}

\section{L'éthos migratoire" des Slaves}

62 À première vue, et à l'opposé des thèses officielles roumaines et albanaises, «le phénomène migratoire est profondément ancré dans l'histoire sociale des peuples et nations de Yougoslavie ${ }^{63}$. Les migrations des Slaves ont eu un caractère exceptionnel : ces migrations se divisent en deux grandes catégories, les grandes migrations des $\mathrm{VI}^{\mathrm{e}}$ et $\mathrm{VII}^{\mathrm{e}}$ siècles et ce que Jovan Cvijić appelle les migrations métanastasiques.

Les grandes migrations des Slaves ont réussi à transformer fondamentalement l'image ethnique de la péninsule : le superstrat ethnoculturel des immigrants s'imposa sur le territoire de la Yougoslavie : ce dernier processus a été favorisé par plusieurs facteurs : la raréfaction des populations autochtones due soit à des épidémies soit à des invasions antérieures (on retrouve ici la thèse de la désertification) et le caractère massif, durable et renouvelé de la colonisation slave ${ }^{64}$.

Les contacts inter-ethniques avec les autochtones concernent essentiellement les populations romanisées et les populations de langue albanaise. Ce dernier cas constitue notamment un enjeu historique, idéologique et politique important d'autant que la dynamique des contacts entre Slaves et Albanais lors des grandes migrations slaves s'est fortement complexifiée à la suite des invasions ottomanes et des migrations métanastasiques qui ont suivi. 
65 qu'il a forgée pour décrire les migrations qui ont résulté des effets de l'invasion ottomane depuis le xive siècle (essentiellement un mouvement migratoire sud-nord et est-ouest, de la part de tous les groupes nationaux «presque constamment en mouvement $\|^{65}$ ), migrations à la suite desquelles la répartition des éléments ethniques a été modifiée dans des proportions considérables dans la péninsule balkanique. Cvijić et son équipe se sont attachés, à partir de 1896, à déterminer dans leurs plus infimes détails ces courants migratoires métanastasiques et leurs résultats ethniques. Certains épisodes historiques sont connus et font partie de la "mémoire nationale" du peuple serbe :

On connaît l'épisode popularisé par l'image et la littérature sous le nom d'“exode des Serbes" : trente mille familles originaires des vallées du Vardar, du Drin, de la Haute-Morava et du Timok, craignant les représailles des Turcs vainqueurs en 1690, suivirent, avec le patriarche Tcharnoievitch, les armées impériales en retraite et allèrent s'installer en Voïvodine, où elles retrouvèrent de nombreux colons serbes et se laissèrent organiser par l'Empire en milices garde-frontières ou granitchari. Ces émigrés assainirent le Banat et fondèrent Novi-Sad, l'“Athènes serbe", tandis que les Turcs et les Albanais se précipitaient sur leurs foyers déserts et transformaient en steppes asiatiques l'ancien centre culturel du vieil empire serbe. Ce mouvement de translation vers le nord s'étant poursuivi, le sultan Mustapha III supprima, le 13 septembre 1766, le patriarchat serbe de Petch en le replaçant sous l'autorité de Constantinople. ${ }^{66}$

Nous avons mentionné ce passage car, au-delà des rappels historiques, il adhère par son vocabulaire même à l'idéologie nationale serbe ${ }^{67}$. D'autre part ce passage illustre les effets des mouvements métanastasiques: renforcement réciproque voire rajeunissement ou "régénération" des premiers établissements serbes par de nouveaux migrants chassés injustement par des Barbares, mais gardant la mémoire de leurs lieux d'origine et de leurs généalogies.

Jovan Cvijić estimait que ces modèles de migrations étaient intimement liés au développement de la conscience nationale après la destruction de l'État médiéval serbe suite à la bataille de Kosovo. Après une avancée turque jusque devant Vienne, et leur défaite en 1683, les Autrichiens pénètrent dans la péninsule balkanique, jusque dans le Kosovo, où des populations serbes les soutiennent. Ils quitteront le Kosovo avec la retraite autrichienne en 1690, épisode connu sous le nom de Velika Seoba (Grande migration). Mais cette population, «en abandonnant les contrées où s'étaient déroulés les faits les plus glorieux de l'histoire serbe pour passer dans la Hongrie méridionale (...), [apporta ses] traditions historiques, un sentiment national très vif et une haine profonde des Turcs ", et composa la population des Confins militaires et de la future Voïvodine $e^{68}$, autrement dit, "après la bataille de Kosovo (...) se forma une puisante conscience nationale serbe, qui, grâce surtout aux migrations, se répandit même en dehors des régions appartenant aux États serbes du Moyen Âge ${ }^{69}$.

L'expression employée ci-dessus par Mousset, "mouvement de translation vers le nord" est très parlante : elle montre bien que les migrations métanastasiques aboutissent, par leur nature collective et organisée (villages entiers, sous la direction des popes orthodoxes), à une "duplication" (une "transplantation"), ethno-religieuse et linguistique, territoriale permettant de maintenir un lien affectif puissant entre les 
nouveaux espaces de colonisation et les anciens berceaux d'origine : les discontinuités spatiales et temporelles sont annulées par la continuité de la mémoire (c'est là qu'intervient le rôle fondamental joué par les traditions, les chants populaires, la religion orthodoxe).

André Blanc affirme que "toute l'histoire des tribus slaves connues est celle des "changements d'habitat" sous l'action de circonstances fortuites et extérieures $»^{70}$, tandis que l'historien Ivo Banac écrit: «l'histoire des Balkans est l'histoire des migrations - pas seulement des peuples, mais aussi des territoires $»^{71}$.

- la question des autochtones : elle est dépendante dans un premier temps de la définition que l'on adopte. Par exemple, le linguiste Rado L. Lenček, dans son ouvrage sur la langue slovène, analysant l'impact linguistique des migrations slaves, définit logiquement comme autochtones les langues pré-existant à l'arrivée des Slaves.

71 De son côté, la récente loi slovène sur les minorités protège uniquement les minorités "autochtones" c'est-à-dire les Italiens et les Hongrois. Les peuples des ex-Républiques de Yougoslavie (Serbes, Croates, etc.) ne sont pas considérés comme des autochtones ${ }^{72}$. De la même façon, certains extrémistes croates estiment que les Serbes établis en Croatie depuis plus de 400 ans ne sont que des résidents temporaires.

On peut également mentionner le cas de la nouvelle constitution macédonienne qui ne reconnaît pas l'existence des Serbes, alors que ceux-ci formaient environ 2,1 \% de la population au recensement de 1981, pourcentage plus élevé que celui des Musulmans, des Tsiganes et des Valaques pourtant reconnus.

73 Dans un article récent consacré à la mosaïque ethnique des Balkans à l'époque des invasions barbares, Nikolay Altankov indique que la ré-implantation de populations était largement pratiquée comme moyen d'assujettir les peuples conquis, et que cette stratégie était utilisée par les gouvernants byzantins et par les envahisseurs barbares. Surtout, Altankov estime qu'il n'y a aucune preuve d'un anéantissement physique de populations autochtones ayant perdu leur position dominante ${ }^{73}$.

74 L'hypothèse du génocide ayant été écartée il reste celles du désert, du refoulement et enfin celle d'un impact des populations pré-slaves.

75 - l'influence des autochtones pré-slaves

D’après Heršak et Mesić, «les Monténégrins ont été fortement influencés par les autochtones des Balkans » et «le substrat paléo-balkanique eut également sa part dans la formation ethnique des Slaves de Macédoine $»^{74}$. Mais quasiment chaque groupe national yougoslave a, peu ou prou, revendiqué une origine non-slave (les Croates, les Macédoniens, les Monténégrins, les Musulmans, les slovènes). Le danger épistémologique ici provient de la facilité qu'il y aurait à expliquer les spécificités régionales et ethniques par la variable "autochtonie" de façon résiduelle, un peu de la même manière qu'André Siegfried introduit la notion de "tempérament ethnique" ou "régional" faute de pouvoir trouver ses facteurs explicatifs dans les variables lourdes traditionnelles.

76 - le refoulement des autochtones: c'est notamment la question des Albanais au Kosovo.

Les Albanais se sont, selon l'historiographie serbe, installés ${ }^{75}$, ou, selon l'historiographie albanaise, rétablis ${ }^{76}$ sur les territoires vidés par la Grande Migration, adoptant l'islam pour les avantages de la classe dominante, chassant de leur terre de nombreux Serbes ${ }^{77}$. En vue de légitimer la possession du Kosovo, de nombreux travaux furent établis, destinés à prouver que l'arrivée des Albanais en Kosovo et en Metohija est une invasion 
"sponsorisée" par les Ottomans. Toutefois, le socialiste serbe Dimitrije Tucović s'est étonné que "la nation balkanique la plus attardée ait été capable d'assimiler ses voisins ", ce qui explique qu'on ait cherché des causes volontaires ${ }^{78}$.

Dans un article datant de 1938, A. Dabinovic relève toutes les hypothèses qui ont été faites concernant l'origine des Albanais (illyrienne, thrace, roumaine), allant jusqu'à se demander si le fait que l'ethnonyme "Albanais" apparaisse seulement lorsque la position sociale de ceux qui le portent, traduisant leur immigration récente, est assurée, il ne traduirait pas une fonction / classe sociale ${ }^{79}$. L'historiographie albanaise défend bien sûr la thèse de la descendance des Illyriens, légitimant l'affirmation d'une présence albanaise au Kosovo avant les Slaves ${ }^{80}$.

D'un côté, le Kosovo est le berceau historique de la Serbie, de l'autre, il représente le lieu de la prise de conscience nationale albanaise, qui s'exprima au travers des revendications de la Ligue de Prizren, en 1878, en réaction aux traités de San Stefano et de Berlin qui attribuaient des territoires albanais à la Serbie et au Monténégro. Le géographe M. Roux établit aussi une distinction dans la conception territoriale des deux acteurs principaux : les Serbes se fonderaient sur une conception historique, leur État doit correspondre à leur Empire du Moyen Âge, les Albanais s'appuieraient sur une conception pragmatique, réclamant leur État où ils sont actuellement majoritaires ${ }^{81}$.

79 - l'hypothèse du désert : cette hypothèse se retrouve à différents niveaux. Il y a bien sûr la thèse déjà mentionnée d'une arrivée des Slaves aux $\mathrm{vI}^{\mathrm{e}}$ et viI ${ }^{\mathrm{e}}$ siècles dans un espace "raréfié" (peste + invasions précédentes ${ }^{82}$ ). Mais il y a également le cas de microdéserts ultérieurs (internes au futur espace étatique serbe puis yougoslave) résultant des invasions ottomanes. Le cas de la Šumadija est le plus net. La région de la Šumadija en Serbie centrale témoigne de processus récents de mouvements d'avancée et de retrait de populations. Suite aux conquêtes ottomanes, la Sumadija fut dépeuplée après le $\mathrm{xv}^{\mathrm{e}}$ siècle, lorsque les ancêtres de la population actuelle cherchèrent refuge dans le haut pays dinarique du sud. La dépopulation était quasi totale. Un voyageur traversant cette région dans la seconde décade $\mathrm{du} \mathrm{xvI}^{\mathrm{e}}$ siècle écrivait que la Šumadija était complètement déserte, tandis qu'un voyageur traversant la même région un siècle plus tôt écrivait avoir vu de nombreux villages et villes. À la fin du xvII siècle et au xvIII ${ }^{e}$ siècle, lorsque ce territoire fut transformé en une forêt dense de chênes, il commença à être repeuplé. Ceux qui alors venait en Šumadija en provenance des régions montagneuses du Dinar, étaient des pionniers défrichant la terre et construisant des maisons en bois.

80 L'expérience migratoire est vitale dans la définition de la structure sociale des villages contemporains de la Šumadija: les hommes de n'importe quelle communauté villageoise sont capables de remonter leur ascendance personnelle jusqu'à l'ancêtre qui s'est établi ici à l'origine et d'établir le groupe descendant patrilinéaire. Les migrations historiques de ce type sont d'une telle importance dans la définition des modèles traditionnels d'implantation et dans la définition des frontières territoriales étatiques, que, nous l'avons vu, une école d'analyse concernée par cette recherche de migrations s'est développée dans l'ethnologie serbe. Ces migrations se sont en partie déroulées avant la formation au XIX siècle d'un État serbe.

81 Ces notions de colonisation, de pionnier, de "frontière", l'emploi des paysans serbes déterritorialisés comme paysans-soldats chargés de défendre l'Empire austro-hongrois contre les attaques ottomanes, sont fondamentales dans le développement de 
l'imaginaire et de la conscience nationales serbes. Il en est de même de l'idée d'une démocratie agraire serbe. Enfin, il faut mentionner également le rôle de la zadruga.

La zadruga est une institution collective patriarchale caractéristique d'une partie des Balkans : cette forme de communauté a été perçue comme un obstacle au processus d'édification étatique et nationale mais en même temps comme le vecteur fondamental de conservation de la conscience ethnique et nationale. Elle a été également "transplantée" lors des mouvements métanastasiques.

Certains intellectuels socialistes serbes ont tenté de montrer le parallèle entre le communautarisme de la zadruga et le socialisme, avançant l'idée selon laquelle la conception serbe de la nation renverrait à une suite continue de cercles concentriques allant du clan à la nation (la nation serbe serait ainsi conçue comme la zadruga ultime).

- la négation de l'autochtonie : on retrouve ici toutes les thèses avancées par les différents acteurs en présence dans l'espace yougoslave pour prouver leur antériorité et leur autochtonie (relative). Nous avons vu plus haut la thèse selon laquelle il n'y aurait que des Serbes : dès lors, les Croates sont des Serbes catholiques, les Bosniaques musulmans, des Serbes islamisés, et les Valaques seraient des Serbes roumanisés qui s'ignorent, etc. De la même façon, les extrémistes croates inversent à leur profit l'argumentation: les Serbes sont des Croates orthodoxes, les Bosniaques musulmans sont des Croates islamisés, et les Slovènes sont des "Croates des montagnes", etc.

\section{Les preuves de la continuité}

La Yougoslavie est une véritable mosaïque ethno-territoriale marquée par de fortes discontinuités internes de l'espace ${ }^{83}$. Ces discontinuités spatiales renvoient en grande partie à des discontinuités historiques (temporelles).

En effet, on peut appliquer à la Yougoslavie ce qu'écrit récemment A. Marès dans un article au titre évocateur («Périodisation de l'histoire et identité nationale chez les Tchèques ») :

À l'image de l'ensemble des peuples d'Europe centrale, les Tchèques ont connu une forte discontinuité étatique, (...) les Tchèques ont reconstruit leur identité au $\mathrm{XIX}^{\mathrm{e}}$ siècle sur quatre fondements. La langue, (...) l'histoire [qui] allait devenir le deuxième pilier d'une identité reconstruite. (...) Troisième pilier: un certain messianisme. (...) Quatrième pilier enfin: l'image en creux renvoyée par l'antimodèle allemand. (...) Conception téléologique qu'ont les Tchèques de leur histoire... ${ }^{84}$

87 Dans le cas de la désintégration yougoslave actuelle, cette reconstruction est le fait des différents peuples constitutifs de l'ex-Yougoslavie. Le but des idéologues croates, serbes, slovènes, bosniaques et autres est de postuler en même temps la singularité et la continuité de leur communauté au-delà des apparents signes de discontinuité. Ici, toutes les sciences sociales sont mobilisables: l'histoire politique et territoriale, la linguistique, l'ethnologie et la science folklorique, etc.

Prenons quelques exemples :

- la Bosnie-Herzégovine :

$\mathrm{Au}$ début de son passage intitulé «la continuité politico-étatique de la BosnieHerzégovine ", l'intellectuel et écrivain croate de Sarajevo, I. Lovrenović écrit :

La Bosnie-Herzégovine, en tant que communauté organisée sur un espace plus ou moins étendu selon la période, mais toujours clairement défini, constitue une création millénaire de l'histoire. Depuis son apogée étatique à la fin du xIv siècle, 
lorsque sous le règne de Tvrtko ${ }^{\text {er }}$ Kotromanic elle constituait le plus important État sud-slave, elle a traversé jusqu'à nos jours des périodes de l'histoire qui lui ont été généralement peu favorables, mais sans jamais perdre tout à fait les caractéristiques de sa physionomie politico-étatique. ${ }^{85}$

- la Croatie, incorporée depuis 1102 dans le royaume de Hongrie puis dans l'Empire austro-hongrois, s'accroche à la permanence de "droits" et à la notion d'une continuité politico-étatique, de façon analogue à celle présentée plus haut pour la BosnieHerzégovine.

Néanmoins, l'entité légale de “Croatie” n’a jamais cessé d'exister sous les Habsbourg. Cela signifiait que la notion de continuité étatique pouvait tôt ou tard devenir un mythe intégrateur. De tels mythes, qui se sont développés en tant que rationalisation d'une faiblesse ou d'une défaite, ont tenu un rôle primordial dans la période d'éveil national - le Kosovo en représentant un dans le cas des Serbes. ${ }^{86}$

En 1864, Eugen Kvaternik définit la Croatie comme s'étendant de l'Isonzo et des Alpes doriques jusqu'à la Drina, à l'Albanie et à Petrovaradin (Danube) ${ }^{87}$. B. Pešelj définit la Croatie ainsi, "géographiquement et historiquement, elle représente dans leur intégrité les pays historiques suivants: Croatie, Slavonie et Dalmatie, qui sont aujourd'hui presqu'entièrement inclus dans le territoire de la République Populaire de Croatie (...). Politiquement et ethnographiquement, la Croatie représente toutes les terres peuplées dans leur majorité par les Croates, en plus des territoires historiques mentionnés cidessus, elle comprend aussi les provinces d'Istrie, de Bosnie-Herzégovine, de Baranja et la partie occidentale de la Bačka" ${ }^{88}$. Ainsi, l'État Indépendant de Croatie recouvrit le territoire yougoslave majoritairement peuplé de Croates $^{89}$.

Pavao Ritter Vitezović, in Croatia rediviva (1700), dans la logique "les Slaves du Sud sont tous des Croates", distingua entre la Croatie blanche (incluant les Slovènes) et la Croatie rouge (Bulgares, Macédoniens, [Monténégrins], Serbes) ${ }^{90}$, de son côté, Ante Starčević fit des Slovènes des Croates de la montagne, les musulmans étant, bien entendu, des Croates islamisés ${ }^{91}$.

- Cette notion de continuité étatique est présente également chez les Serbes avec d'autant plus de force que la Serbie a connu également des États médiévaux développés et s'est rendu indépendante avant la Croatie, la Bosnie-Herzégovine, la Slovénie ou la Macédoine : surtout, la diversité des assises territoriales des diverses formes étatiques de la Serbie permet aux idéologues serbes de "cumuler" tous les cas historiques de discontinuités spatio-temporelles pour justifier les prétentions territoriales d'une Grande Serbie.

Ce qui est très important ici c'est l'importance accordée aux migrations comme "liant" assurant l'homogénéité et la continuité de l'entité serbe, et cela à plusieurs points de vue. Les "justes" causes de ces migrations sont les luttes entre la Chrétienté et les Barbares asiates musulmans : elles expliquent le départ de la Vieille Serbie, berceau historique du Kosovo, l'implantation dans des régions qui vont être de nouveaux berceaux de la nation serbe soit par le développement de la résistance culturelle puis politique, soit du fait que ces Serbes vont jouer le rôle de "tampons" contre l'avancée ottomane (dans les Confins Militaires), renforçant ainsi l'image d'une Serbie comme gardienne des valeurs chrétiennes: les Serbes se perçoivent comme un peuple élu auquel incombe une mission, celle d'être à la fois l'avant-garde et le dernier rempart de la Chrétienté contre les Barbares païens : les Slaves, et notamment les Serbes, sont les "défenseurs" de l'Occident. 

Croatie et la Hongrie, la Croatie étant encore en 1980 le « rempart de la catholicité $»^{92}$. Son intégration au royaume hongrois en 1102 a légitimé une vision occidentaliste de la Croatie, ainsi, "la conscience nationale croate (...) est essentiellement nourrie par l'image idéale du sacrifice du peuple pour le salut de l'Occident chrétien dans sa lutte contre les mécréants ", dans laquelle s'est conservée la mémoire "d'individus "tombés sur les remparts du christianisme" ", idéal peu, ou pas, partagé par les Dalmates ${ }^{93}$. En fait, la Croatie «se veut la Muraille Chrétienne, défendue par les Serbes, suite au recul croate devant les invasions ottomanes $»^{94}$. De même, la Slovénie est vue comme le rempart de l'Europe orientale contre le germanisme ${ }^{95}$.

Après que les Huns aient envahi les territoires slaves et déraciné les tribus slaves de leur sol natal, les tribus serbo-croates, pour éviter un nouvel ouragan mongol, ont abandonné les plaines de Galicie et les franges boisées des Carpathes, et sont apparus sur les frontières orientales de l'empire romain. A leur première apparition sur la scène de l'histoire du monde, la destinée assigna aux Slaves du sud un rôle qu'ils ont loyalement rempli jusqu'à présent. Leur rôle était noble [rappel de l'accord passé avec les empereurs byzantins]. La nation serbo-croate est restée fidèle à ce rôle - notamment être les gardiens et les protecteurs de la civilisation européenne - jusqu'à présent..$^{96}$

Dans ce dernier texte écrit en pleine guerre mondiale et lors de la naissance du Royaume des Serbes, Croates et Slovènes, l'accent est mis sur la nation serbo-croate, mais les Serbes ont longtemps estimé et estiment encore qu'ils ont joué le rôle primordial dans cette mission.

Il est d'ailleurs très intéressant de montrer qu'une fois le danger ottoman disparu, le clivage chrétienté / islam a été remplacé par un clivage Occident/ Orient qui introduisait une frontière culturelle au sein même de la Yougoslavie. Stjepan Radić, leader du Parti républicain paysan croate dans l'entre-deux-guerres, tenait les propos suivants :

Notre histoire, notre situation géographique, notre orientation vers la Hongrie nous font fédéralistes, pour ne point nous mettre dans la dépendance des Balkans qui sont le prolongement de l'Asie. Notre devoir est d'européaniser les Balkans et non de balkaniser Croates et Slovènes, (...) la Yougoslavie ne constitue une nation qu'au point de vue extérieur. (...) De plus, nous sommes, les Serbes et nous, d'une formation mentale totalement étrangère l'une à l'autre. La Serbie fut une caserne héroïque ; nous, qui n'avions même pas notre armée, nous avons été un cabinet de travail. La Serbie est revenue à la vie libre par une insurrection, elle s'est faite et maintenue par des guerres ininterrompues. Nous avons conservé une vie nationale par un travail culturel méthodique, par un effort patient d'intellectuels. Nos journaux et nos livres ont été nos seuls fusils pendant un siècle de luttes, sous une domination étrangère, contre l'Autriche, la Hongrie et l'Italie. Cette œuvre toute cérébrale nous a amenés à la compréhension du slavisme. La vie militaire du peuple frère, élevé autrement, l'a laissé à l'étape du serbisme... Nous avons dépassé ce stade, car nous ne faisons pas chez nous de croatisme. ${ }^{97}$

Ces considérations seront reprises aujourd'hui par les dirigeants slovènes et croates. En Yougoslavie, il existe une littérature en provenance des régions occidentales de ce pays (Slovénie et Croatie) tendant à placer la frontière Occident / Orient au sein même de la Yougoslavie, privilégiant d'un côté les Républiques du nord-ouest, anciennes possessions "progressistes" des Habsbourg, catholiques et / ou protestantes, au détriment des Républiques "balkaniques", anciennes possessions de l'Empire ottoman, orthodoxes et / ou musulmanes ${ }^{98}$. 
99 En 1990, Franjo Tudjman affirme qu'en raison de leurs expériences historiques, les Croates et les Slovènes doivent constituer des États souverains alliés dans le cadre de la Communauté Européenne et que ces deux peuples, d'un côté, et les Serbes de l'autre, appartiennent à deux civilisations complètement différentes ${ }^{99}$.

\section{La quête des origines linguistiques}

100 Les idéologues de la Slovénie et de la Macédoine, espaces moins bien dotés que les autres Républiques d'une continuité politico-étatique, ont misé sur l'argumentation linguistique pour prouver l'ancienneté de leurs communautés. Ils sont partis à la recherche d'un dialecte fondateur ${ }^{100}$.

101 Dans le chapitre d'un livre intitulé "Nos ancêtres les Vénètes“, Matej Bor ${ }^{101}$ propose la thèse selon laquelle le peuple et la langue slovènes seraient issus des anciens Vénètes. Cet auteur utilise des inscriptions en vieux vénète remontant au Vème siècle avant J.C., procède à une reconstruction de certaines caractéristiques de ce dialecte et établit un parallèle avec le vieux slave/slovène. Cette démarche est très proche de celle des idéologues roumains soucieux de renforcer la seule continuité des Daces au détriment de la romanité. Ici, l'appel aux Vénètes permet de prendre des distances par rapport aux autres peuples slaves (serbes et croates) et à mieux singulariser l'ethnicité slovène.

102 Cependant, certains auteurs estiment que la méthodologie historico-comparative employée par Bor n'est pas linguistiquement et archéologiquement fondée et concluent que l'effort de Bor pour affirmer une "ethnogénèse" vénète du slovène renvoie plus à une relecture romantique visant à renforcer un sentiment national qu'à une œuvre scientifique ${ }^{102}$.

103 L'exemple du vieux macédonien est également intéressant : là aussi, comme en Slovénie (et ailleurs), les revendications nationalistes surdéterminent largement les argumentations linguistiques.

104 Selon Henrik Birnbaum ${ }^{103}$, l'expression “vieux bulgare", qui ne désigne pas sa sphère d'application fonctionnelle initiale, est une appellation justifiée d'un point de vue ethnolinguistique dans la mesure où les deux premiers prédicateurs des Slaves, Cyrille et Méthode, bien que locuteurs natifs de Grèce, maîtrisaient clairement, dès leur jeune âge, le dialecte bulgare du sud-ouest qui était parlé dans les environs de Salonique. Pour Birnbaum, l'expression “vieux macédonien”, introduite dans les années récentes, principalement par des chercheurs macédoniens (de Yougoslavie), doit être considérée comme anachronique car on ne peut pas parler d'un langage macédonien séparé, qui aurait été essentiellement différent du bulgare précoce, en usage dès le IX siècle.

Les différences que l'on peut trouver entre l'école littéraire d'Ohrid et la scripta en usage dans la capitale de la Bulgarie, Preslav, ne justifient aucunement l'existence de deux langues séparées.

Et Birnbaum conclut en affirmant que si "vieux bulgare" est un synonyme légitime pour "vieux slavon d'Église", il n'est pas justifié d'introduire le terme concurrent et reconstruit de "vieux macédonien".

Il s'agit là d'une question controversée qui, malheureusement, est biaisée par les sensibilités nationales. Quand on sait l'importance historique, religieuse et idéologique du vieux slave d'Église et le renouveau actuel de la "question macédonienne", on comprend mieux la nature des enjeux . 
Les deux exemples mentionnés ci-dessus témoignent du souci de justifier, à partir d'un acte fondateur (langue, héros, mythe, etc.), une continuité temporelle. On peut également, tout en restant dans le cadre d'une argumentation basée sur des critères linguistiques, tenter de prouver et justifier une continuité spatiale: on peut mentionner par exemple les travaux sur l'existence d'une continuité linguistique slave (les différentes langues slaves ne seraient que les dialectes d'une même langue originelle et, dès lors, il serait nécessaire de les ré-unifier, dans le cadre d'un panslavisme linguistique). Enfin, il est nécessaire de rappeler ici les enjeux idéologiques et politiques de la construction puis de la déconstruction du serbo-croate (ou croatoserbe) et la place prééminente accordé au dialecte štokavski.

Vuk St. Karadžić est considéré comme le créateur de l'idéologie grand-serbe, via le vecteur de la langue. S'il a confié la "serbité" à tous les locuteurs du štokavski ${ }^{104}$, c'est en partie en reprenant une erreur commise par des linguistes qui l'ont précédé. Le premier à avoir considéré le štokavski comme une langue serbe est l'historien allemand Ludwig von Schlötzer (1735-1809), erreur perpétuée par des slavistes tchèques, slovaques et slovènes (Josef Dobrovský, Jan Kollar, Pavel Josef Šafařik, Jernej Kopitar, Franc Miklošić), tandis que le kajkavski ne se vit attribuer comme locuteurs que des Slovènes ${ }^{105}$, et que seul le čakavski fut considéré comme langue croate ${ }^{106}$. Donc, nous avons affaire à une définition de la nation par le facteur linguistique, facteur utilisé par des intellectuels, précurseurs du mouvement national en vue d'édifier, non pas un État, mais une nation (serbe, slovène). Il est vrai que là aussi, les effets linguistiques des mouvements métanastasiques doivent être pris en compte ${ }^{107}$.

\section{Conclusion}

Parlant du cas yougoslave, Jim Seroka écrit: "Nous avons vu un groupe de nations devenir captives d'une série de mythes historiques dysfonctionnels $»^{108}$.

Ces mythes sont ceux de la continuité prouvée par tous les moyens y compris ceux d'un cumul des discontinuités. C'est également celui de la singularité nationale.

Selon Stoiše Naumov, les ethnogénèses officielles des divers pays balkaniques font de chaque nation une entité unique qui n'a pas son pendant, ainsi,

les Serbes forment le plus ancien peuple du monde,

les Albanais constituent un phénomène ethnolinguistique sans pareil sur la planète,

les Roumains sont des frères de sang exclusifs des Italiens, des Français et des

Espagnols,

les Macédoniens sont les descendants directs d'Alexandre le Grand,

les Grecs n'ont rien à voir avec les Balkans hormis leur situation géographique

et les Bulgares doivent, en bons Tatars, s'étendre en Asie ou au moins jusqu'à la

Volga.

113 L'auteur, principalement intéressé par la "question macédonienne", conclut que "manifestement, la macédoine de légumes trouve son pendant dans la ratatouille grecque: les deux sont composées de très nombreux ingrédients, les deux ont été cuisinées pendant des siècles et les deux vous empoisonnent l'existence $"{ }^{109}$.

114 Le thème de l'unicité est le propre de toute idéologie nationaliste : c'est ainsi que depuis plusieurs siècles, la construction d'une identité nationale nippone a été fondée sur la "singularité" absolue attribuée à la culture japonaise. 
Déjà, au XIV ${ }^{\mathrm{e}}$ siècle, Kitabatake Chikafusa affirme que le Japon est unique et supérieur à tous les autres pays parce que la dynastie autochtone est la seule à avoir une origine divine et à avoir régné sans interruption depuis "l'âge des dieux". Cette idée sera reprise cinq cents ans plus tard par les idéologues militaristes des années 30 qui en feront le fondement de la tradition nationale. Le courant historiographique dominant est donc le particularisme en contraste avec les théories chronologiques bouddhiques qui se veulent universelles. En outre, comme on le verra, l'identité culturelle (à travers l'idée d'esprit ou de tempérament national) se fonde sur la perpétuation d'éléments immuables et ôte toute importance aux changements, pourtant admis par les anciens historiens. ${ }^{110}$

On pourrait multiplier les exemples tirés de contextes sociaux, spatiaux et temporels très différents. Cette recherche obstinée et irrationnelle de la "singularité absolue" aboutit à ce que les parties en présence, après avoir sélectionné à leur avantage certains faits historiques, linguistiques ou ethnologiques, présente des légitimités territoriales conflictuelles sans être pour autant contradictoires dans le temps.

Les habitats antérieurs d'un peuple ne doivent aucunement justifier des prétentions politiques et territoriales actuelles et encore moins les génocides et autres "purifications ethniques".

117 Aujourd'hui, les tentatives de réaménagement constitutionnels d'États multiethniques font une place importante à la notion de "peuples fondateurs", de "peuples historiques" et / ou de "peuples constitutifs" comme partenaires à égalité. Mais cette évolution qui semble synchroniquement la plus respectueuse de la pluralité ethnique, recouvre des cas de figures historiques différents : il y a ainsi une différence entre le désir des peuples autochtones du Canada (longtemps opprimés et absents de la scène politique) en montée "ascendante" et celui des Serbes de Croatie qui, refusant de devenir des minorités, demandent à continuer d'être considérés comme un des "peuples constitutifs" de Croatie ou de Bosnie-Herzégovine

La solution consistant à considérer les différents peuples des nouvelles Républiques comme des "peuples fondateurs" constitutifs de ces Républiques permettrait de tirer un trait sur leurs ethnogénèses conflictuelles mais supposerait une remise à zéro de leur mémoire collective. Or cela est contradictoire avec les logiques nationalistes actuelles qui poussent à l'ethnicisation de la politique.

\section{NOTES}

1. Le fait que les "poussées" nationalistes aient pu être portées par des acteurs sociaux différents est soit niée, soit, version plus subtile, récupérée avec l'argument d'une continuité de la nation prouvée par la diversité historique même de ces acteurs sociaux. On pourrait faire la distinction entre un inter-classisme synchronique et un inter-classisme diachronique.

2. «C'est un mythe que de considérer les nations comme un moyen naturel, donné par Dieu, de classer les hommes et de considérer les nations comme une destinée politique naturelle même si leur venue est tardive » (Gellner (Ernest)., Nations et nationalisme, Paris : Payot, Gellner, 1989, p. 76). 
3. On peut aussi utiliser l'idéologie de la réification pour construire une image "naturelle" et permanente des systèmes de domination; par exemple présenter le groupe dirigeant comme une "vérité universelle" ou un "peuple élu" illustre l'idée de réification.

4. Pour reprendre le titre d'un ouvrage co-dirigé par E. Hobsbawm et T. Ranger (Hobsbawm (Eric), Ranger (Terence), eds., The invention of tradition, Cambridge : Cambridge University Press, 1983).

5. Titre du livre de B. Anderson (Anderson (Benedict), Imagined communities. Reflections on the origin and spread of nationalism, London : Verso, 1983).

6. Ce couple renvoie à d'autres couples tels que quantitatif / qualitatif, unicité / universalité.

7. Cerquiglini (Bernard), La naissance du français, Paris : PUF, coll. Que-Sais-Je, 1991, p. 25.

8. Par exemple le berceau préhistorique et le berceau historique des Serbes n'est pas le même.

9. Melman (Billie), "Claiming the nation's past : the invention of an Anglo-Saxon tradition ", in Reinharz (Jehuda), Mosse (George L.), eds., The impact of western nationalisms. Essays dedicated to Walter Z. Laqueur on the occasion of his $70^{\text {th }}$ birthday, London : Sage, 1992, p. 221.

10. Nous pensons que, au-delà des terminologies employées, il existe des "invariants".

11. Cf. parmi les travaux d'Anthony Smith, "National identity and myths of ethnic descent", Research in Social Movements, Conflict and Change, 7, 1984 ; The ethnic origins of nations. Oxford: Basil Blackwell, 1986 ; National identity, London : Harmondsworth, 1991 ; «Chosen peoples : why ethnic groups survive », Ethnic and Racial Studies, 15 (3), july 1992.

12. Le mot "provisoirement" signifie que, dans des contextes de crise et / ou de transition, cette question des origines ethniques peut très bien être actualisée, manipulée par les hommes politiques et les intellectuels.

13. Bonte (Pierre), Izard (Michel), éds., Dictionnaire de l'ethnologie et de l'anthropologie, Paris : PUF, 1992, p. 248.

14. Voir par exemple l'importance de ces thèmes dans les différentes Histoires publiées par les Éditions Horvath (Albanie, Roumanie, peuples ouraliens, etc.), histoires rédigées par des personnalités scientifiques des ex-régimes d'Europe de l'est.

15. Dans un article publié en 1991, A. Simic consacre un passage intitulé: "South Slavic ethnogenesis" (Simic (Andrei), "Obstacles to the development of a Yugoslav national consciousness : ethnic identity and folk culture in the Balkans ", Journal of Mediterranean Studies, 1 (1), 1991).

16. Voir Gonzalez (Nancie L.), McCommon (Carolyn S.), eds., Conflict, migration, and the expression of ethnicity, Boulder : Westview Press, 1989 ; Roosens (Eugeen E), Creating ethnicity : the process of ethnogenesis, Newbury Park / London: Sage, 1989; Fardon (Richard), "“African ethnogenesis": limits to the comparability of ethnic phenomena ", in Holy (Ladislav), ed., Comparative anthropology, Oxford : Basil Blackwell, 1987.

17. Les distinctions effectuées par les linguistes entre substrat, adstrat et superstrat renvoient à cette dynamique (linguistique) des contacts multi-ethniques. Comme le dit Hammond dans son ouvrage sur les migrations et les invasions en Grèce et dans les contrées voisines : « dans les deux derniers chapitres nous n'avons pas utilisé le terme à la mode de "substrat" et très peu les concepts de race et d'origine raciale, pour lesquels le mot actuel est "ethnogénèse" » (Hammond (Nicholas G.L.), Migrations and invasions in Greece and adjacent areas, Park Ridge : Noyes Press, 1976, p. 129).

18. Vermeer (Willem), "Albanians and Serbs in Yugoslavia», in Heuvel (Martin van den), Siccama (Jan G.), eds., The disintegration of Yugoslavia, Amsterdam / Atlanta : Rodopi, 1992, pp. 103-104.

19. Ibid., p. 105.

20. Ainsi, par exemple, les définitions française et allemande de la nation, synthétisées dans l'opposition droit du sol et droit du sang, s'inscrivent dans le cadre de l'appropriation de l'AlsaceLorraine. 
21. Pavlowitch (Stevan K.), « Spence (Richard B.), Nelson (Linda L.), eds., Scholar, patriot, mentor : historical essays in honour of Dimitrije Djordjevic », Slavonic Review, 19, 1992.

22. La Borderie (Arthur Lemoyne de), «Éloge historique de Dom Lobineau », in Inauguration du monument élevé à la mémoire de Dom Lobineau, 3 mai 1886. Nantes : Société des Bibliophiles Bretons et de l'Histoire de Bretagne, 1886, p. 33. "Mais ce sentiment de la solidarité nationale ne peut naître et se fortifier que par la connaissance de la nation et de son existence antérieure, de son passé et de son présent, de sa tradition et de sa destinée ; il se développe d'autant plus que cette connaissance est plus complète et que la génération présente peut mieux apprécier la gloire, la vertu, la grandeur de celles qui l'ont précédée ».

23. Gotsev (Dimitar), « Bulgarian scholars and their role in the national liberation movement of the Bulgarians in Macedonia during the late $19^{\text {th }}$ and $20^{\text {th }}$ centuries ", Macedonian Review, 14 (1), 1991, p. 30.

24. Macedonian Review, 14 (1), 1991, p. 8.

25. «Mais qu'est-ce que la "vérité scientifique" ? Je pense que ceux qui parlent de "vérité scientifique" appartiennent à la catégorie de pensée des sociétés pré-sécularisées, dont les croyances sont fondées sur des dogmes religieux non remis en question, pas sur la pensée rationelle. » (à propos de l'expression employée "vérité scientifique", Magocsi (Paul Robert), "The birth of a new nation, or the return of an old problem? The Rusyns of East Central Europe ", Canadian Slavonic Papers, 34 (3), september, 1992, p. 204).

26. Martis (Nicolaos K.), The falsification of Macedonian history, Athens: Graphic Arts of Athanassiades Bros., 1985.

27. Borza (Eugene N.), In the shadow of Olympus. The emergence of Macedon, Princeton: Princeton University Press, 1990, p. 91.

28. Lunt (Horace G.), « Notes on nationalist attitudes in Slavic studies », Canadian Slavonic Papers, 34 (4), december 1992, p. 459.

29. Ibid., p. 467.

30. Ibid., p. 470.

31. Jelavich (Charles), «Serbian textbooks : toward greater Serbia or Yugoslavia?», Slavic Review, 42 (4), winter 1983, p. 619.

32. Seton-Watson (R. W.), Serbia yesterday, to-day, and to-morrow. A school address published for the Kossovo Day Committee, Westminster : Vacher and Sons Ltd, s.d. (1916), p. 7.

33. Ibid., p. 8.

34. Chaboseau (A.), Les Serbes et leur épopée nationale, (préface de Milenko R. Vesnitch, ministre du "Royaume-Uni des Serbes, Croates et Slovènes", membre de l'Institut de France), Paris : Éditions Bossard, 1919, p. 7.

La question du Kosovo est une question complexe: pour deux bilans récents opposés, voir Tomashevich (George), "The problem of Kosovo through the centuries: a historical and anthropological overview ", Australian Slavonic and East European Studies, 4 (1-2), 1990 (pro-serbe) et Vermeer (Willem), art.cit. (pro-albanais). Voir également le livre de T. Emmert plus centré sur la bataille de Kosovo, sa mémorisation et ses usages politiques (Emmert (Thomas A.), Serbian Golgotha: Kossovo, 1389, Boulder : East European Monographs (278), 1990). Voir également Roux (Michel), Les Albanais en Yougoslavie. Minorité nationale, territoire et développement, Paris : Maison des Sciences de l'Homme, 1992.

35. Zirojevic (Olga), «Les premiers siècles de la domination étrangère », in Le Kosovo-Metohija dans l'histoire serbe, Lausanne : L'âge d'homme, 1990, p. 70.

36. Pipa (Arshi), "The other Albania : a Balkan perspective », in Pipa (Arshi), Repishti (Sami), eds., Studies on Kosova, Boulder : East European Monographs (155), 1984, p. 241.

37. Dragnich (Alex N.), Todorovich (Slavko), The saga of Kosovo, Boulder: East European Monographs (170), 1984, pp. 29-44. 
38. Ducellier (Alain), « Genesis and failure of the Albanian state in the fourteenth and fifteenth centuries ", in Pipa (Arshi), Repishti (Sami), eds., op.cit., p. 4.

39. Cf. Wilkinson (H. R.), Maps and politics. A review of the ethnographic cartography of Macedonia, Liverpool : University Press, 1951.

40. Grimm (Gerhard), "Ethnographic maps of the Kosova region from 1730 to 1913 », in Pipa (Arshi), Repishti (Sami), eds., op.cit., p. 47.

41. Cf. Hammel (Eugene), Halpern (Joel), « Observations on the intellectual history of ethnology and other social sciences in Yugoslavia ", Comparative Studies in Society and History, 11, 1969, ainsi que le numéro double de la revue Etnološki Pregled (23-24), 1988, consacré à l'histoire de l'ethnologie en Serbie, en Croatie, en Slovénie, en Bosnie-Herzégovine et en Macédoine.

42. Pavković (Nikola), Bandić (Dušan), Kovačević (Ivan), « Aspirations and directions of the developement of ethnology in the Socialist Republic of Serbia (1945-1983) », Etnološki Pregled, (23-24), 1988, p. 9.

43. «La méthode anthropogéographique a efficacement contribué à l'étude d'importantes questions concernant les influences mutuelles entre l'homme et son environnement naturel, puis à propos de l'origine et des migrations des habitants » (ibid., p. 8).

44. Ibid.

45. Voir les travaux de Jovan Cvijić (Remarques sur l'ethnographie en Macédoine, Paris: Georges Roustan, 1907; "The zones of civilization of the Balkan peninsula », Geographical Review, 5 (6), june 1918; La Péninsule balkanique. Géographie humaine, Paris: Armand Colin, 1918 ; «Des migrations dans les pays yougoslaves : l'adaptation au milieu », Revue des Études Slaves, 3 (1-2), 1923 ; «Des migrations dans les pays yougoslaves : leurs conséquences », Revue des Études Slaves, 3 (3-4), 1923).

46. Cf. Lederer (Ivo J.), Yugoslavia at the Paris Peace Conference: a study in frontier making, New Haven / London : Yale University Press, 1963, pp. 126-128, qui cite notamment la participation de Cvijić ainsi que d'autres ethnologues "yougoslaves".

47. Ibid., p. 93. Et Lederer ajoute "le travail le plus célèbre de Cvijic, La péninsule balkanique. Géographie humaine (Paris, 1918), lui a valu de l'occident une large attention et des acclamations. Mais ces précédents travaux sur la Macédoine et le Serbie étaient aussi connus et admirés. Voir Cvijiceva Knjiga (Belgrade, 1927); Iz uspomena i zivota (Novi Sad, 1923) ; P. Slepcevic, Jovan Cvijic (Sarajevo, 1927) ». Et plus loin « bien qu'il ait étroitement collaboré avec d'autres ethnologues à la conférence de la paix - J. Radonic, A. Belic, S. Mihaldjic, N. Zupanic, et avec les historiens L. Vojnovic and F. Sisic - la plupart du travail collectif aboutit à des conclusions reprenant celles des travaux de Cvijic menés entre 1895 et 1914 ».

48. Dans sa monographie sur la Macédoine, T. R. Georgevitch mentionne avec admiration les travaux de Cvijić mais avec la réserve suivante: " pour que la chose soit tout à fait claire, nous citerons ici quelques-unes des raisons données par M. Cvijic. Mais afin que l'on ne puisse nous accuser de partialité, nous ne retiendrons des arguments de M. Cvijic, que ceux auxquels on ne saurait reprocher d'être l'expression du patriotisme serbe actuel, et qui, par conséquent ne peuvent faire l'objet d'aucune discussion »! (Georgevitch (T.R.), La Macédoine, Paris : Bernard Grasset, 1919, p. 16).

49. Magaš (Branka), The destruction of Yugoslavia. Tracking the break-up 1980-92, London / New York : Verso, 1993.

50. Varady (Tibor), Dimitrijevic (Nenad), « Ex-Yugoslavia », East European Constitutional Review, 2 (4), fall 1993 and 3 (1), winter 1994.

51. Ibid.

52. Carroll (Terrance), "Owners, immigrants and ethnic conflict in Fiji and Mauritius », Ethnic and Racial Studies, 17 (2), april 1994, p. 302.

53. Thucydide, CEuvres complètes, Paris : Gallimard, coll. Bibliothèque de la Pléiade, 1964, p. 694.

54. Ibid. 
55. Deletant (Dennis), «Rewriting the past: trends in contemporary Romanian historiography », Ethnic and Racial Studies, 14 (1), 1991.

56. La première romanité, ou romanité urbaine, n'aurait pas pu résister, sauf exceptions, à l'assimilation ethnique.

57. Cf. Schramm (Gottfried), Eroberer und Eingesessene. Geographische Lehnnamen als Zeugen der Geschichte Südeuropas im 1. Jahrtausend n. Chr. Stuttgart : Hiersemann, 1981 ; Schramm (Gottfried), «Frühe Schicksale der Rumänen - Acht Thesen zur Lokalisierung der lateinischen Kontinuität in Südosteuropa ", Zeitschrift für Balkanologie XXII/1, XXII/2 (1986) et XXIII/1 (1987); ainsi que Schütz (Istvan), « Les huit thèses de Gottfried Schramm et l'ethnogénèse roumaine ", Travaux de Linguistique et de Philologie, 30, 1992.

58. Ruzé (Alain), Ces Latins des Carpathes. Preuves de la continuité romaine au nord des Carpathes, Berne / Francfort s. Main : Peter Lang, 1989.

59. Latour (Éliane de), Les temps du pouvoir, Paris : Éditions de l'École des Hautes Études en Sciences Sociales, vol. 3, 1992, p. 25.

60. Bonkalo (Alexander), The Rusyns, Boulder : East European Monographs (293) [traduction en anglais du livre original paru en 1940 à Budapest sous le titre A rutének].

61. Ces questions mériteraient, ainsi que celles des bases ethniques et religieuses des migrations rurales / urbaines, d'être mieux analysées dans le cas yougoslave notamment.

62. Pour celui de l'ethnogénèse proposée par les idéologues du mouvement breton en France, voir Guillorel (Hervé), «Surdéterminants socio-culturels du conflit linguistique: langue, religion et politique. Le cas de la Bretagne », in Guillorel (Hervé), Hartweg (Frédéric), éds., Langues et conflits, Nanterre : I.P.I.E., 1987.

63. Hersak (Emil), Mesic (Milan), "L'espace migratoire de Yougoslavie: historique des migrations yougoslaves ", Revue Européenne des Migrations Internationales, 6 (2), 1990, p. 27.

64. Diverses théories ont été proposées sur la dynamique des migrations successives des peuples slaves. On mentionnera au passage l'hypothèse selon laquelle les Serbes et les Croates, à l'instar des Bulgares, auraient été des tribus non slaves qui se seraient imposées à des premiers migrants slaves et auraient adopté leur langue tout en leur imposant leurs ethnonymes.

65. Cvijic (Jovan), «Les Mouvements métanastasiques dans la Péninsule des Balkans », Monde Slave, 1, juillet 1917, p. 86.

66. Mousset (Albert), Le royaume des Serbes, Croates et Slovènes, Paris : Éditions Bossard, 1921, p. 90.

67. Les travaux publiés à la fin du XIX ${ }^{\text {ème }}$ siècle et au début du XX ${ }^{\text {ème }}$ siècle par les intellectuels français sur les Balkans, sur la Serbie et sur la Yougoslavie sont fortement pro-serbes (rôle de l'anti-germanisme, défiance à l'égard de la Turquie, rôle de la Serbie dans la première guerre mondiale, etc.). D'où une valorisation extrême des qualités et des valeurs serbes et de l'amitié franco-serbe.

68. Cvijic (Jovan), « Les mouvements métanastasiques » (art.cit.), pp. 94-95.

69. Cvijic (Jovan), La Péninsule balkanique... (op.cit.), p. 165.

70. Blanc (André), Géographie des Balkans, Paris : PUF (coll. Que sais-je), 1971.

71. Banac (Ivo), The national question in Yugoslavia. Origins, history, politics. London: Cornell University Press, 1984, p. 33.

72. À cet égard la loi hongroise sur les minorités est plus tolérante car elle définit comme "autochtones" les minorités établies sur son territoires depuis plus de 100 ans.

73. Altankov (Nikolay), "The Balkans in the seventh century : an ethnic approach », in Spence (Richard B.), Nelson (Linda L.), eds., Scholar, patriot, mentor : historical essays in honour of Dimitrije Djordjevic, Boulder : East European Monographs (320), 1992.

74. Hersak (Emil), Mesic (Milan), art.cit., p. 29.

75. Ibid., p. 36. 
76. Veinstein (Gilles), «Les provinces balkaniques (1606-1774)", in Mantran (Robert), éd., Histoire de l'Empire ottoman, Paris : Fayard, 1989, p. 320.

77. Cvijic (Jovan), op.cit., p. 150.

78. La thèse de l'albanisation des Serbes est de moins en moins courante, mais on en trouve encore des exemples. Pour une critique de cette théorie, cf. Roux (Michel), op.cit., pp. 419-420.

79. Dabinovic (A.), « Early Balkan migration », Slavonic Review, 16 (47), janvier 1938, p. 410.

80. Biberaj (Elez), « Kosovë : The Struggle for recognition », London : Conflict studies, (137/138), The Albanian problem : two views, 1982, p. 24.

81. Roux (Michel), "La crise de la Kosova: histoire d'une intolérance réciproque", L'ethnographie, 85, 2 (106), automne 1989, p. 135 ; Rusinow (Dennison), « Nationalities policy and the "national question" ", in Ramet (Pedro), ed., Yugoslavia in the 1980's, Boulder : Westview press, 1985, p. 145. Signalons enfin que Krbovčko Polje, là où se produisit la chute des Croates en 1493 devant les Turcs est le pendant croate de Kosovo, et Knin, "la capitale des rois croates".

82. Cf. Hersak (Emil), Mesic (Milan), art.cit., p. 28. J. Reinach a pu écrire que les migrations furent de « paisibles conquêtes où les Slaves n'avaient qu'à remplir les vides de l'ouest dépeuplé » (Reinach (J.), La Serbie et le Monténégro, Paris : Calmann-Lévy, 1876, p. 5). A. Lyall écrit même que " quand en 568, les Lombards migrent en Italie des plaines panoniennes, l'espace vide qu'ils ont laissé derrière eux ont été remplis par des arrivants slaves des Carpathes » (Lyall (Archibald), "The making of modern Slovenia ", Slavonic Review, 17 (50), january 1938, p. 404).

83. Cf. la carte intitulée "discontinuités internes de l'espace yougoslave" in Foucher (Michel), éd., Fragments d'Europe. Atlas de l'Europe médiane et orientale, Paris : Fayard, 1993, p. 190.

84. Marès (Antoine), « Périodisation de l'histoire et identité nationale chez les Tchèques ", Revue des Études Slaves, 66 (1), 1994, pp. 192-193.

85. Lovrenovic (Ivan), « Les Croates et la Bosnie », Messager Européen, 7, 1993, p. 118.

86. Trifkovic (Srdjan), « The first Yugoslavia and origins of Croatian separatism », East European Quarterly, 26 (3), fall 1992, p. 346.

87. Warnier (Raymond), « Ouvrages sur la Croatie », Monde slave, 15, juin 1938, p. 475.

88. Peselj (Branko M.), «Contemporary Croatia in the Yugoslav federation : its constitutional status and socio-economic position », Journal of Croatian studies, 2, 1961, p. 80.

89. Ibid., p. 81.

90. Sirc (Ljubo), "The national question in Yugoslavia », South Slav Journal, 9 (1-2), spring, 1986, p. 82 ; Banac (Ivo), op.cit., p. 73 ; Samardzic (Radovan), «Les idées du siècle des Lumières et l'éveil national des peuples yougoslaves", in Les Lumières et la formation de la conscience nationale chez les peuples du sud est européen, Bucarest: Association internationale d'études du sud-est européen (actes du colloque Paris, 11-12/04/68), 1970, p. 109. Toutefois, les appellations Croatie blanche et Serbie blanche font référence à des premiers États, le premier établi au dessus des Carpathes, le second entre l'Elbe et la Saale (Dvornik (Francis), Les Slaves, histoire et civilisation de l'Antiquité aux débuts de l'époque contemporaine, Paris: Seuil, 1970, p. 36; Djuric (Ivan), "Les racines historiques du conflit serbo-croate », Etudes, (4), octobre 1991, p. 293).

91. Sirc (Ljubo), art.cit., p. 82 ; Banac (Ivo), op.cit., p. 87.

92. Clarté (Abel), « Les Croates », Les dossiers de l'histoire, (27), septembre 1980, pp. 30, 34.

93. Djuric (Ivan), art.cit., p. 295.

94. Djuric (Ivan), «Les Serbes, la Serbie et la crise yougoslave », Conférence Paris IV - Sorbonne, 16/05/94.

95. Martel (René), « La Slovénie et les problèmes politiques contemporains », Monde slave, 3 (5), mai 1926, p. 278.

96. Savic (Vladislav R.), The reconstruction of south-eastern Europe, London: Chapman and Hall, 1917, pp. 31-32.

97. Cité in Pezet (Ernest), Simondet (Henri), La Yougoslavie en péril ?, Paris : Bloud et Gay, 1933, p. 39. 
98. Cf. Bakic-Hayden (Milica), Hayden (Robert M.), «Orientalist variations on the theme "Balkans" : symbolic geography in recent Yugoslav cultural politics », Slavic Review, 51 (1), spring 1992. Cf. aussi «Zagreb», diffusé sur Arte, le 06/09/94, dans lequel, outre les euphémismes, le commentaire fait état d'Occident croate et d'Orient serbe.

99. Le Monde, 02/10/90.

100. Cf. Guillorel (Hervé), " De l'utilisation politique de la variété dialectale ", in Guillorel (Hervé), Sibille (Jean), éds., Langues, dialectes et écriture. Les langues romanes de France, Paris / Nanterre : I.E.O. / I.P.I.E., 1993.

101. Bor (Matej), «Die Venetische Sprache », in Savli (Jozef), Bor (Matej), Unsere Vorfahren die Veneter, Dunaj : Veneti, 1988.

102. Lenček (Rado L.), Gjurin (Velemir), «Borove jezikoslovne premise njegove venetske teorije ", Slavistična Revija, 38 (4), octobre 1990.

103. Birnbaum (Henrik), «The linguistic type of old church slavonic viewed in the perspective of time and space ", Scando-Slavica, 36, 1990.

104. Le serbo-croate est une langue subdivisée en trois dialectes (štokavski, kajkavski et čakavski) sur la base de l'isoglosse -quoi- (što/šta - kaj - ča), le dialecte štokavski formant la base littéraire, c'est celui-ci qui est dénommé serbo-croate. Lui même comprend trois sub-divisions (parlers) selon la prononciation de la lettre disparue - jat - (ekavski, ijekavski et ikavski). Aucune de ces divisions et sub-divisions ne correspond à des clivages nationaux ou religieux, mais plutôt à des variations régionales.

105. Jacques Ancel aussi estime que le kajkavski est une langue slovène (Ancel (Jacques), Peuples et nations des Balkans, Paris : CTHS, 1992 [1930], p. 172), rattacher le slovène au kajkavski permet d'affirmer que la langue slovène est une branche du serbo-croate (comme l'affirme Sirc (Ljubo), art.cit., p. 81).

106. Banac (Ivo), "The Confessional "Rule" and the Dubrovnik Exception: The origins of the "Serb-Catholic" Circle in nineteenth-century Dalmatia ", Slavic Review, 42 (3), fall 1983, p. 451.

107. Pour une synthèse récente des questions relatives aux langues slaves parlées en Yougoslavie, voir Thomas (Paul-Louis), «Serbo-croate, serbe, croate..., bosniaque, monténégrin : une, deux..., trois, quatre langues ? ", Revue des Études Slaves, 66 (1), 1994.

108. Seroka (Jim), "Yugoslavia: marching to the beat of a different drummer ", History of European Ideas, 19 (1-3), 1994, p. 495.

109. Naumov (Stoise), " Macédoine et ratatouille », Makedonsko Delo, in Courrier international, (188), 9-15/06/94, p. 16.

110. Picone (Mary), «Le nouveau nationalisme japonais. Construction d'une mémoire collective », Genre Humain (Le), (18), automne 1988, p. 121.

\section{RÉSUMÉS}

Les espaces en voie de structuration/destruction étatique et nationale sont régulièrement le lieu d'élaboration, d'invention, par des groupes sociaux spécifiques de mythes et/ou d'Histoires visant à prouver une continuité étatique ou nationale sur une longue période. Ces productions idéologiques contiennent toujours une problématique sur la dialectique "autochtonie/ migrations » et proposent des "ethnogénèses » censées rendre compte des contacts entre les 
différents groupes ethniques qui se sont succédés au sein d'un même territoire. Cet article propose une première typologie de ces contacts et l'illustre sur la base de l'espace yougoslave.

Within territories concerned by state and nation-building/destroying processes, specified social groups have always created, invented myths and/or Histories with the purpose of proving a state and national continuity over the long time. These ideological products always include considerations about the dialectical relationships within the couple autochtony/migration(s) and offer ethnogenesis which are supposed to describe and explain the dynamics of contacts between all the ethnic groups which have been established within the same territory. This article based upon a simple typology of contacts, illustrates it by the Yugoslavian instance.

\section{AUTEURS}

\section{HERVÉ GUILLOREL}

Hervé Guillorel est chercheur au C.N.R.S

\section{PATRICK MICHELS}

Patrick Michels est titulaire d'un DEA de Politique Comparée. 